\title{
Numerus clausus: le «test d'aptitudes pour les études de médecine» (AMS) permet-il de trouver les personnes les plus aptes?
}

\author{
K.-D. Hänsgen, B. Spicher
}

Deutsch erschienen

in Nr. 31/2002
Correspondance:

Prof. Dr. Klaus-Dieter Hänsgen Université Fribourg Département de psychologie Zentrum für Testentwicklung und Diagnostik Route Englisberg 9 CH-1763 Granges-Paccot

\section{Résumé}

Depuis 1998, les Universités de Bâle, Berne, Fribourg et Zurich appliquent un numerus clausus (NC) aux études de médecine et utilisent un test d'aptitudes comme critère d'admission. Quelles sont les expériences faites jusqu'ici? L'admission repose-t-elle réellement sur l'aptitude? Il a été possible, pour la première fois en Suisse, d'examiner ces questions à l'aide des données empiriques fournies par deux volées (1998 et 1999) en comparant les résultats obtenus aux examens propédeutiques à ceux du «test d'aptitudes pour les études de médecine» (AMS). Le résultat du test (ci-après: test) permet de prévoir avec précision tant la durée des études que les notes d'examen: les personnes qui obtiennent en priorité une place sont celles qui achèvent leurs études le mieux et le plus rapidement. A l'intérieur des sous-groupes également (définis en fonction de la langue, l'âge, le sexe, la discipline, l'université), un même test signifie un même pronostic quant aux chances de réussir l'examen: le principe de l'égalité de traitement est ainsi respecté.

Par rapport à d'autres critères d'admission, l'AMS reste sans concurrence non seulement parce qu'il est économique, non-discriminatoire et impartial mais aussi parce qu'on a prouvé qu'il sélectionne les candidats en fonction de l'aptitude à étudier la médecine.

\section{Introduction}

Conformément aux bases légales cantonales relatives au NC, l'admission aux études de médecine doit reposer sur l'aptitude, qui se définit, quelle qu'en soit la formulation, par la concordance entre capacités personnelles et exigences. Pour déterminer l'aptitude, il faut:

- préciser, en fonction des exigences, sur quoi l'aptitude doit porter. Il est pertinent de s'en tenir à l'aptitude aux études car le manque de capacité concerne ces dernières et c'est lui qu'il s'agit de gérer équitablement. L'aptitude à exercer la profession serait par exemple trop difficile à définir quant aux exigences, tant les disciplines et les activités médicales sont variées, même si ces deux aptitudes ne sont bien sûr pas indépendantes l'une de l'autre. - concevoir des critères d'admission qui permettent de prévoir à la fois les chances de réussite d'une personne et de distinguer les personnes en fonction de leurs aptitudes. Un critère d'admission ne se justifie que s'il est scientifiquement prouvé que ce sont les plus aptes qui ont réellement le plus de chance d'être admis aux études.

Deux critères sont déterminants pour évaluer l'aptitude aux études: les places d'études doivent être accordées en priorité à ceux qui finissent leurs études

1. le plus rapidement possible (durée optimale), et

2. le mieux possible (qualité des résultats), soit en ayant acquis des compétences et des habiletés de haut niveau.

En effet, en prévenant le prolongement des études, voire les abandons, on ne met pas les ressources - déjà restreintes - inutilement à contribution et on les libère plus rapidement pour un nouvel étudiant. Pour les études de médecine, deux facteurs par exemple en limitent l'accès: le fait qu'on ne peut augmenter le nombre de patients des hôpitaux universitaires à volonté ainsi que des places de stages et de laboratoire aux installations coûteuses. Il ne faut toutefois pas en rester à cette perspective purement économique car les étudiants ont aussi intérêt à limiter les abandons après une ou deux années, afin de perdre le moins de temps possible avant de pouvoir exercer leur métier.

Il ne s'agit pas non plus de confondre aptitude et penchant. La notion de penchant est plutôt à mettre en relation avec l'intérêt pour les professions médicales. L'idéal serait que les candidats aux études soient suffisamment informés pour que leurs attentes face aux études et à leur future profession soient réalistes. Comme dans de nombreux autres domaines, l'intérêt ne peut être ici assimilé à la capacité ou à l'aptitude. 
Figure 1

Moyenne $(\diamond)$ et écart-type du test pour les deux volées en fonction du $1^{\text {er }}$ propédeutique.

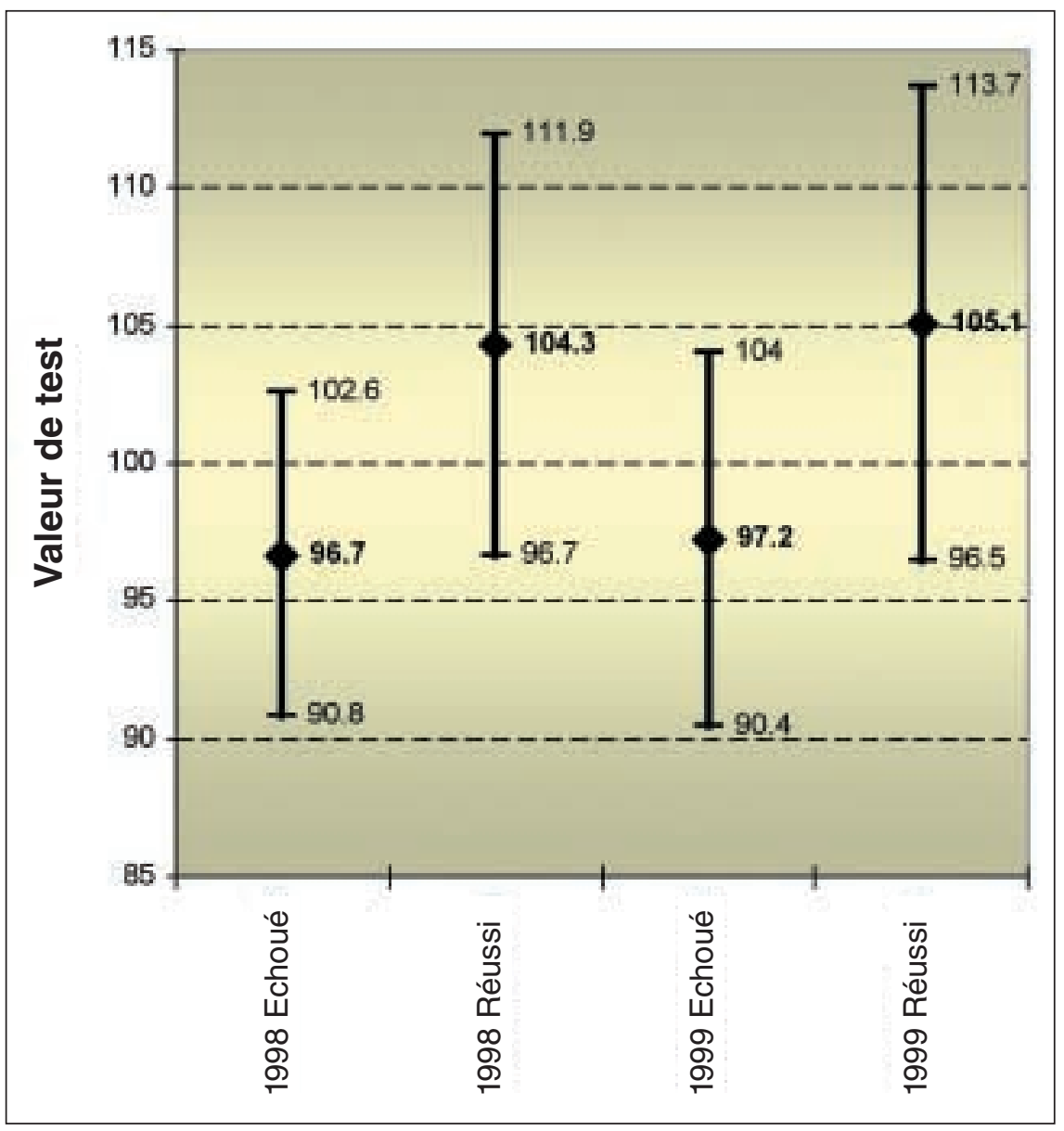

* Nous tenons ici à remercier vivement Messieurs Megert et Neuhaus ainsi que Madame Hirvi de l'OFSP pour leur précieuse collaboration.
La Suisse utilise un test d'aptitudes (AMS) comme critère d'admission. L'AMS a fait l'objet d'un rapport de Hänsgen et Spicher [1, 2] qui analysent les expériences faites jusqu'ici. L'AMS se fonde réellement sur l'aptitude si on peut prouver que 1 . les corrélations entre test AMS et réussite à l'examen prédisent la durée des études et 2 . celles entre test AMS et notes d'examen prédisent la qualité des études. L'AMS est une adaptation du test allemand pour les études de médecine (TMS), dont la qualité des pronostics relatifs à la réussite des études est avérée (voir notamment [3]).

La relation entre test et examen est analysée pour les candidats qui ont participé au test en 1998 et 1999. En 1998, le numerus clausus était obligatoire pour la médecine humaine, en 1999 pour la médecine humaine et vétérinaire aux Universités de Bâle, Berne, Fribourg et Zurich. Les personnes immatriculées en 1998 ont eu jusque-là quatre possibilités de se présenter aux examens, la variante optimale consistant à avoir étudié deux ans jusqu'au $2^{\mathrm{e}}$ propédeutique. Pour les personnes immatriculées en 1999, elles n'ont pu se présenter au $1^{\text {er }}$ propédeutique qu'en été ou automne 2000.

L'Office fédéral de la santé publique (OFSP) a recensé les données relatives aux examens*. Le principal critère d'évaluation de l'AMS réside dans la possibilité de prédire le succès à l'examen à partir du test. Comme on le sait, l'AMS vise à prédire l'aptitude aux études et non à mesurer l'état des connaissances. Il vérifie l'aptitude du candidat à élaborer des connaissances (à partir de textes, par ex.), à reconnaître et à appliquer des règles logiques, ou encore à se concentrer et à mémoriser. Les propédeutiques ont au contraire pour objectif de vérifier l'état des connaissances effectivement acquises au cours des études (compétences, puis plus tard habiletés). Le bilan présenté ici est une version condensée du rapport édité par Hänsgen et Spicher en 2001 [4].

\section{Test et $1^{\text {er }}$ propédeutique}

Les moyennes du test obtenues par les groupes définis en fonction du passage du $1^{\text {er }}$ propédeutique sont exposées dans le tableau 1, la durée des études (une ou deux années jusqu'à l'examen) n'ayant ici que peu d'influence.

Le groupe «examen réussi du $1^{\mathrm{er}}$ coup» se distingue nettement de tous les autres groupes avec une moyenne au test significativement plus élevée. Les personnes qui n'ont réussi qu'à leur $2^{\mathrm{e}}$ tentative ou qui n'ont pas encore essayé arrivent à une moyenne avoisinant 100, tandis que les personnes qui ont raté une ou deux fois l'examen ont une moyenne nettement inférieure à 100 .

Les tests des personnes qui se sont présentées au $1^{\mathrm{er}}$ propédeutique ont été distingués en fonction du résultat obtenu à cet examen (fig. 1). On retrouve pour les deux volées des différences significatives assez semblables.

Le rapport entre le résultat au test et la probabilité de réussir l'examen dessine une courbe on ne peut plus linéaire (fig. 2): plus le résultat au test est élevé, plus le candidat a des chances de réussir l'examen, ce qui signifie aussi qu'en dessous d'un certain résultat, le candidat n'a pratiquement aucune chance de réussir son examen. Il est ainsi prouvé qu'en attribuant à ce groupe des places d'études devenues vacantes suite à des abandons, on fait baisser le taux de réussite général d'une volée. 
Figure 2

Probabilité de réussite au $1^{\text {er }}$ propédeutique par test, résumée pour les deux volées 1998 et 1999.

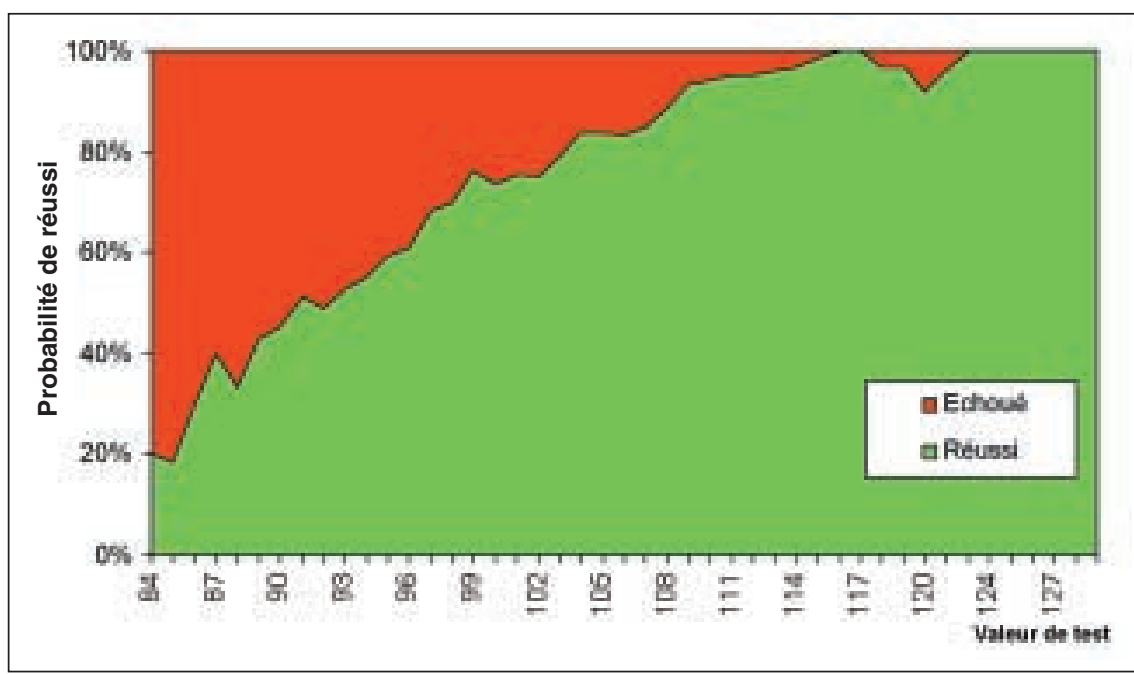

\section{Effet de l'admission de personnes inscrites sur la liste d'attente}

Les places devenues vacantes en raison d'abandon sont attribuées à des personnes dont le résultat au test se situe en dessous du critère de sélection (soit la valeur du test déterminée par la capacité d'accueil). Ce critère est fixé chaque année en fonction de la seule capacité d'accueil: il ne distingue donc pas du tout les personnes aptes des inaptes. Si le nombre de places disponibles diminue, le critère sera un résultat au test plus élevé, à l'inverse si le premier augmente, le second baissera. Il ressort du tableau 2 que 63 personnes, qui n'avaient en fait pas atteint le critère et ont été admises à partir de la liste d'attente, se sont présentées au $1^{\text {er }}$ propédeutique. Leurs chances de réussir l'examen ne sont que de $40 \%$ alors qu'elles se montent à $78 \%$ pour les personnes ayant obtenu un résultat au test su-

Tableau 1

Moyenne (m) et écart-type (s) du test pour les groupes définis en fonction du $1^{\mathrm{er}}$ propédeutique; entre les lignes doubles: homogénéité de plusieurs moyennes; $\mathrm{n}$ : nombre de personnes.

\begin{tabular}{|c|c|c|c|c|c|c|c|c|c|}
\hline & \multicolumn{3}{|c|}{$\begin{array}{l}\text { Test } 1998 \\
\text { (après } 2 \text { ans) }\end{array}$} & \multicolumn{3}{|c|}{$\begin{array}{l}\text { Test } 1999 \\
\text { (après } 1 \text { an) }\end{array}$} & \multicolumn{3}{|c|}{ Total } \\
\hline & $\mathbf{n}$ & m & s & $\mathbf{n}$ & m & s & $\mathbf{n}$ & m & $\mathbf{s}$ \\
\hline raté deux fois & 46 & 97,2 & 6,4 & 11 & 95,9 & 7,1 & 57 & 96,9 & 6,5 \\
\hline raté une fois & 47 & 96,3 & 5,4 & 172 & 97,3 & 6,8 & 219 & 97,1 & 6,6 \\
\hline pas encore essayé & 33 & 99,6 & 6,9 & 83 & 98,3 & 7,7 & 116 & 98,6 & 7,5 \\
\hline réussi à la $2^{\mathrm{e}}$ tentative & 101 & 99,9 & 5,4 & 30 & 101,7 & 7,9 & 131 & 100,3 & 6,1 \\
\hline réussi du $1^{\text {er }}$ coup & 373 & 105,5 & 7,7 & 370 & 105,4 & 8,6 & 743 & 105,4 & 8,1 \\
\hline
\end{tabular}

périeur au critère. Seules deux personnes se sont présentées au $2^{\mathrm{e}}$ propédeutique. On peut donc s'attendre à ce que ces différences s'accentuent au cours des études.

Ces constatations mettent en évidence les conséquences qu'auraient une augmentation de la capacité d'accueil sans qu'on assiste à une modification du comportement des candidats: allongement de la durée des études, diminution des chances de succès, et probablement multiplication des abandons.

On devrait réfléchir aux moyens d'inciter davantage de personnes aptes à étudier la médecine à déposer leur candidature, autrement dit se demander si les facteurs dissuasifs n'effrayeraient pas les «mauvaises» personnes et donc sélectionneraient à mauvais escient. L'AMS est un instrument qui permet de limiter l'admission aux études en fonction de la capacité d'accueil et sur la base de l'aptitude à étudier. Les universités ne risquent donc plus d'être surchargées et les personnes admises ont de bonnes chances de réussir leurs études. Si l'on suppose qu'un nombre croissant de maturités devrait susciter un intérêt croissant pour les études de médecine, il ne reste que des facteurs dissuasifs pour expliquer l'intérêt, certes toujours élevé, mais stagnant, pour la faculté de médecine. Une partie de ces facteurs sont d'ordre professionnel. Mais si davantage de personnes envisageraient le test comme une chance de déterminer leur aptitude (et de vérifier leur penchant pour de tels études), il y aurait plus de personnes aptes qui entameraient cette filière.

\section{Egalité des chances entre les différents sous-groupes}

Le critère d'admission doit remplir une autre exigence-clé: l'égalité des chances. Autrement dit, une même aptitude doit offrir les mêmes chances de succès indépendamment de l'âge, du sexe, de l'origine, etc. Si l'on compare les tests des différents sous-groupes définis en fonction du $1^{\mathrm{er}}$ propédeutique, on s'aperçoit primo que quel que soit le sous-groupe, la moyenne de ceux qui l'ont réussi se distingue nettement de celle des personnes qui ont échoué, et secundo que les moyennes des premiers divergent peu. Le pouvoir prédictif du test est donc universel. Dans le tableau 3 figurent les moyennes ainsi que les écarts-types du test. Il faut noter que les probabilités de réussir ne sont pas les mêmes à l'intérieur des sous-groupes. En effet, le rapport entre les plus aptes et les moins aptes diffère d'un groupe à l'autre. 
Tableau 2

Succès lors des deux propédeutiques et critère (valeur du test déterminée par la capacité d'accueil): pour plus de détails, se reporter au texte.

\begin{tabular}{|c|c|c|c|c|}
\hline \multicolumn{5}{|l|}{$1^{\text {er }}$ propédeutique } \\
\hline & & échoué & réussi & $\mathbf{n}$ \\
\hline \multirow[t]{2}{*}{ résultat au test inférieur au critère } & nombre & 38 & 25 & 63 \\
\hline & en $\%$ & $60,3 \%$ & $39,7 \%$ & \\
\hline \multirow[t]{2}{*}{ résultat au test supérieur au critère } & nombre & 238 & 849 & 1087 \\
\hline & en $\%$ & $21,9 \%$ & $78,1 \%$ & \\
\hline \multicolumn{5}{|l|}{$2^{e}$ propédeutique } \\
\hline & & échoué & réussi & n \\
\hline \multirow[t]{2}{*}{ résultat au test inférieur au critère } & nombre & 1 & 1 & $2(!)$ \\
\hline & en $\%$ & $50,0 \%$ & $50,0 \%$ & \\
\hline \multirow[t]{2}{*}{ résultat au test supérieur au critère } & nombre & 51 & 293 & 344 \\
\hline & en $\%$ & $14,8 \%$ & $85,2 \%$ & \\
\hline
\end{tabular}

Le pouvoir prédictif n'est pas spécifique à l'université. Dans toutes les universités, il existe un écart de 5 points au minimum entre les groupes définis en fonction de l'examen. On trouve toutefois des variations dans le taux de réussite: elles ne sont manifestement pas (seulement) dues à une rigueur différente aux examens, mais aussi à des prestations personnelles hétérogènes (sont ici à prendre en considération «profils de candidat» variables d'une université à l'autre et conséquences des transferts d'étudiants). La situation particulière de Fribourg apparaît ici clairement: c'est elle qui compte le plus grand nombre d'étudiants transférés. Augmenter davantage les capacités reviendrait probablement à admettre encore plus de personnes ayant peu de chance de réussir leurs examens.

Envisage-t-on les groupes définis en fonction de l'examen par rapport à l'appartenance sexuelle, un même intervalle sépare les tests des deux groupes. Il n'a pas été possible de démontrer qu'il existait en 1998 et 1999 une différence significative entre les résultats au tests des deux sexes, bien que les hommes aient tendance à avoir de meilleurs résultats au test (environ 1,5 point). Les résultats au test sont pratiquement identiques pour les deux groupes qui ont réussi l'examen, ceux des groupes qui ont échoué n'ont que
0,4 point d'écart. C'est la preuve que les variations possibles entre les sexes au niveau des résultats au test ne sont pas à interpréter comme une inégalité des chances mais sont très probablement dues à une répartition inégale entre femmes et hommes, puisque les femmes sont sur-représentées. Les «chances» de réussir le $1^{\text {er }}$ propédeutique sont pratiquement les mêmes pour une même aptitude, ce qui prouve que toute crainte d'une inégalité de traitement entre les sexes est infondée.

Nous avons pu montrer que jusqu'à présent les résultats au test des personnes plus âgées qui ont passé tardivement leur maturité sont chaque année inférieurs à ceux de leurs contemporains qui ont passé leur maturité le plus tôt possible. Pour ce faire, nous avons formé deux groupes de «personnes âgées» (nées avant 1975): celles qui ont eu leur maturité après 1995 («maturité tardive») et celles qui l'ont eu avant 1996 («maturité précoce»). Ces groupes ont été comparés avec les «personnes jeunes». Dans les trois groupes, les tests permettent de distinguer nettement ceux qui ont réussi de ceux qui ont échoué au $1^{\mathrm{er}}$ propédeutique (au moins un écarttype). Il est possible de comparer la réussite du groupe des personnes âgées «maturité tardive» avec les autres groupes, même si elles ont obtenu des tests inférieurs, ce qui tendrait à prouver qu'une plus grande «expérience de vie» devient un atout si «l'expérience scolaire» a été rafraîchie en passant récemment les examens de maturité.

Quant aux trois groupes linguistiques, ils présentent des différences comparables en fonction du succès à l'examen, même si pour le groupe italophone elle est un peu moins marquée (à noter que ce groupe est aussi le plus restreint). C'est un résultat important puisqu'il prouve que pour un même test il n'existe aucune différence d'aptitude, quel que soit le groupe linguistique. L'égalité des chances est garantie entre les groupes linguistiques.

Malgré des taux de réussite variés, on arrive à la même conclusion en ce qui concerne les deux disciplines de la médecine (humaine et vétérinaire). Les personnes qui réussissent dans les deux disciplines le font avec des résultats presque identiques, il en va de même pour celles qui échouent. 
Tableau 3

Moyenne (m) et écart-type (s) du test pour les groupes définis en fonction du $1^{\text {er }}$ propédeutique.

\begin{tabular}{|c|c|c|c|c|c|c|}
\hline \multirow[t]{2}{*}{ université } & \multicolumn{3}{|l|}{ échoué } & \multicolumn{3}{|l|}{ réussi } \\
\hline & $\mathbf{n}$ & m & $s$ & n & m & s \\
\hline Berne & $46(16 \%)$ & 98,0 & 6,2 & $235(84 \%)$ & 104,2 & 7,8 \\
\hline Bâle & $48(21 \%)$ & 97,1 & 5,4 & $186(79 \%)$ & 102,6 & 8,2 \\
\hline Fribourg & $69(40 \%)$ & 93,8 & 6,2 & $104(60 \%)$ & 102,3 & 9,0 \\
\hline Zurich & $113(25 \%)$ & 98,6 & 6,7 & $347(75 \%)$ & 106,8 & 7,4 \\
\hline sexe & échoué & & & réussi & & \\
\hline hommes & $100(20 \%)$ & 96,8 & 7,0 & $407(80 \%)$ & 104,6 & 8,3 \\
\hline femmes & $176(27 \%)$ & 97,2 & 6,3 & $467(73 \%)$ & 104,8 & 7,9 \\
\hline âge et maturité & échoué & & & réussi & & \\
\hline nés avant 1975, maturité avant 1996 & $15(44 \%)$ & 96,5 & 4,7 & $19(56 \%)$ & 105,7 & 8,1 \\
\hline nés avant 1975, maturité après 1995 & $7(16 \%)$ & 94,9 & 5,1 & $37(84 \%)$ & 101,1 & 6,0 \\
\hline nés après 1974 & $254(24 \%)$ & 97,1 & 6,7 & $816(76 \%)$ & 104,8 & 8,1 \\
\hline langue & échoué & & & réussi & & \\
\hline allemand & $216(21 \%)$ & 97,1 & 6,3 & $806(79 \%)$ & 104,8 & 8,1 \\
\hline français & $41(53 \%)$ & 95,9 & 7,5 & $37(47 \%)$ & 104,1 & 7,3 \\
\hline italien & $19(38 \%)$ & 98,3 & 6,7 & $31(62 \%)$ & 102,7 & 7,7 \\
\hline discipline & échoué & & & réussi & & \\
\hline médecine humaine & $124(27 \%)$ & 96,9 & 7,2 & $336(73 \%)$ & 105,3 & 8,8 \\
\hline médecine vétérinaire & $59(48 \%)$ & 97,9 & 6,0 & $64(52 \%)$ & 104,4 & 6,8 \\
\hline
\end{tabular}

\section{Résultat au test et $2^{\mathbf{e}}$ propédeutique}

Jusqu'à présent, seules les personnes de la volée 1998 ont été en mesure de passer le $2^{\mathrm{e}}$ propédeutique. Elles devaient auparavant avoir réussi le $1^{\mathrm{er}}$ propédeutique. Les personnes ayant passé le test de justesse sont ici sous-représentées, elles ne seront plus nombreuses que ces prochaines années, ce qui mettra à disposition davantage de résultats pour le $2^{\mathrm{e}}$ propédeutique. On peut cependant déjà constaté que les résultats au test des personnes qui l'ont réussi se distinguent nettement de ceux des personnes qui ont échoué (tableau 4).

Le test a un bon pouvoir prédictif aussi bien pour le $2^{\mathrm{e}}$ propédeutique que pour $1^{\mathrm{er}}$ propédeutique. C'est ce que révèle la comparaison des corrélations bisériales entre test et examen. Cette re-

Tableau 4

Moyenne $(\mathrm{m})$ écart-type $(\mathrm{s})$ du test $d^{\prime}$ après le $2^{e}$ propédeutique

\begin{tabular}{|llll|}
\hline $\mathbf{2}^{\mathrm{e}}$ propédeutique & $\mathbf{n}$ & $\mathbf{m}$ & $\mathbf{s}$ \\
\hline échoué & 52 & $\mathbf{1 0 2 , 2}$ & $\mathbf{6 , 6}$ \\
\hline réussi & 294 & 106,0 & 7,6 \\
\hline
\end{tabular}

lation est exprimée par le coefficient d'association «Eta» qui a une valeur de 0,42 pour le $1^{\text {er }}$ propédeutique et de 0,40 pour le $2^{\mathrm{e}}$. Mais si l'on compte toutes les personnes qui auraient dû se présenter à ce dernier examen comme «propédeutique échoué», l'«Eta» est même de 0,44.

\section{Test et notes à I'examen ( ${ }^{\mathrm{er}}$ propédeutique)}

A côté de la prédiction de la durée des études, il existe un deuxième critère d'évaluation important: la prédiction de la qualité des études. Cette analyse est rendue difficile en raison de l'hétérogénéité des systèmes d'évaluation qui diffèrent d'une université à l'autre et d'une année à l'autre.

Le $1^{\text {er }}$ propédeutique a été passé à Berne en 1999 (en partie), et à Bâle, Fribourg et Zurich en 1999 et 2000 dans quatre branches. Nous avons retenu la dernière note au cas où l'examen a été passé deux fois.

Les relations ont été saisies au moyen des coefficients de corrélation. Entre 0,30 et 0,40, la valeur prédictive est considérée comme élevée en comparaison avec d'autres tests, et au-dessus 
Tableau 5

Corrélation du test et des points aux sous-tests avec les notes du $1^{\text {er }}$ propédeutique («Reform 2» à Berne et moyenne de Bâle, Zurich et Fribourg).

\begin{tabular}{|c|c|c|c|c|c|c|c|}
\hline & $\begin{array}{l}\text { ensemble } \\
\text { des examens } \\
\text { oraux } \\
\text { (Berne) }\end{array}$ & $\begin{array}{l}\text { ensemble } \\
\text { des examens } \\
\text { à choix multiples } \\
\text { (Berne) }\end{array}$ & $\begin{array}{l}\text { physique } \\
\text { et } \\
\text { physiologie }\end{array}$ & $\begin{array}{l}\text { chimie } \\
\text { et } \\
\text { biochimie }\end{array}$ & $\begin{array}{l}\text { biologie générale } \\
\text { et } \\
\text { humaine I }\end{array}$ & $\begin{array}{l}\text { biologie générale } \\
\text { et } \\
\text { humaine II }\end{array}$ & moyenne \\
\hline Total &, 55 &, 59 &, 51 & 49 &, 50 &, 48 &, 55 \\
\hline médecine humaine & & &, 50 &, 47 &, 48 &, 44 &, 53 \\
\hline médecine vétérinaire & & &, 55 &, 57 & 60 & ,66 &, 66 \\
\hline
\end{tabular}

Tableau 6

Corrélation entre les notes au $1^{\mathrm{er}}$ propédeutique et le test à l'intérieur des différents sous-groupes (toutes les corrélations sont significatives au seuil de 1\%).

\begin{tabular}{|c|c|c|c|c|c|c|}
\hline & $\mathbf{n}$ & $\begin{array}{l}\text { physique et } \\
\text { physiologie }\end{array}$ & $\begin{array}{l}\text { chimie et } \\
\text { biochimie }\end{array}$ & $\begin{array}{l}\text { biologie générale } \\
\text { et humaine I }\end{array}$ & $\begin{array}{l}\text { biologie générale } \\
\text { et humaine II }\end{array}$ & moyenne \\
\hline \multicolumn{7}{|l|}{ université } \\
\hline Berne (1999: en partie) & 64 & 64 &, 51 &, 48 &, 58 &, 59 \\
\hline Bâle & 233 & ,39 &, 35 & ,30 &, 38 & ,41 \\
\hline Fribourg & 169 &, 50 &, 53 &, 38 &, 39 &, 53 \\
\hline $\begin{array}{l}\text { Zurich } \\
\text { âge et maturité }\end{array}$ & 458 & ,52 &, 49 & ,51 & ,50 &, 58 \\
\hline né avant 1975, maturité avant 1996 & 30 & ,50 &, 58 &, 52 & 43 &, 59 \\
\hline né avant 1975, maturité après 1995 & 34 & 41 &, 34 &, 45 &, 31 &, 46 \\
\hline $\begin{array}{l}\text { né après } 1974 \\
\text { sexe }\end{array}$ & 859 &, 47 &, 44 &, 45 &, 46 &, 52 \\
\hline hommes & 411 & 46 &, 40 &, 43 &, 41 &, 49 \\
\hline $\begin{array}{l}\text { femmes } \\
\text { langue }\end{array}$ & 512 &, 50 &, 48 &, 47 & ,50 &, 56 \\
\hline allemand & 829 &, 47 &, 43 &, 45 &, 46 &, 52 \\
\hline français & 52 &, 57 &, 55 &, 46 &, 50 &, 60 \\
\hline italien & 42 & ,41 &, 42 & ,41 &, 50 &, 47 \\
\hline
\end{tabular}

de 0,40 comme très élevée. Les corrélations des résultats au test des deux années sous revue avec les notes du $1^{\mathrm{er}}$ propédeutique sont élevées pour toutes les branches et pour la moyenne. Même les crédits d'examen accordés désormais à Berne présentent une très haute corrélation avec le résultat au test et ses différentes variables. Ce résultat est aussi à mettre au compte d'une grande variation, et donc par là même à une évaluation plus différenciée des personnes.

On peut constater qu'il existe une grande corrélation entre les notes et les résultats au test pour les deux disciplines, même si pour la médecine vétérinaire elle aurait tendance à être un peu plus grande. Cela démontre que le test prédit la qualité des études dans les deux disciplines.

D'autres sous-groupes sont comparés dans le tableau 6. La qualité des pronostics que permet le test pour les notes d'examen ne dépend pas de l'université: le type d'examen - «essai écrit», «choix multiple écrit», «oral» - n'a aucune influence sur la qualité du pronostic (voir Hänsgen et Spicher [4]). De même, il n'existe aucun indice que celle-ci varierait en fonction du sexe, de la langue ou de l'âge, preuve supplémentaire que le principe de l'égalité des chances est respecté. 


\section{Conclusions relatives à la détermination de I'aptitude}

La présente évaluation permet de préciser, pour la durée des études jusqu'au $2^{\mathrm{e}}$ propédeutique de médecine, certains acquis concernant la relation entre admission sur la base d'un test d'aptitudes et aptitude:

- Le test d'aptitudes pour les études de médecine (AMS) porte réellement sur l'aptitude aux études, car les personnes obtenant de meilleurs résultats au test 1 . ont une plus grande probabilité de réussir leurs examens plutôt que celles qui ont de moins bons résultats au test et 2 . font des études d'une plus grande qualité, exprimée par de meilleures notes à l'examen. Le rapport entre résultat au test et aptitude aux études se situe à un niveau statistiquement significatif qui justifie l'utilisation du test comme critère d'admission.

- L'AMS satisfait également au critère d'égalité des chances. A aptitude égale (même test), on a fondamentalement les mêmes chances de réussir l'examen quel que soit son sexe, sa langue, sa discipline, son âge ou son université. Seul le groupe des plus âgés avec une maturité tardive présente cette particularité que même les résultats au test moins élevés ont une forte probabilité de réussir l'examen, alors que dans tous les autres groupes les personnes qui réussissent se distinguent des personnes qui échouent.

- $2^{\mathrm{e}}$ propédeutique: bien que peu de personnes aient tenté de le passer, nous avons suffisamment d'indices pour affirmer que le pouvoir prédictif du test est aussi élevé pour le $1^{\text {er }}$ que pour le $2^{\mathrm{e}}$ examen.

- Les universités peuvent désormais admettre les étudiants en fonction de leurs capacités, ce qui améliore les conditions dans lesquelles les études se font. C'est l'avantage le plus important de l'AMS car il en justifie l'usage. Il permet en outre de décider équitablement qui peut étudier dans l'université de son choix et qui doit accepter d'étudier ailleurs.

- Ce qui manifestement ne change pas, c'est le nombre des personnes aptes qui s'inscrivent pour étudier la médecine. Le numerus clausus (NC) est un moyen de sélection «doux» puisque seuls quelque $20 \%$ des candidatures sont refusées. Une partie des $80 \%$ des person- nes admises ne commence pas les études, la principale cause étant le transfert dans une autre université. Ce phénomène a pour double conséquence que les places libérées sont attribuées à des personnes moins aptes, soit celles qui appartiennent aux 20\% des résultats inférieurs au test et donc que le niveau d'aptitude des étudiants ne diffère guère de celui d'une situation sans NC. Il est prouvé qu'admettre des personnes moins aptes (simplement pour exploiter toutes les places disponibles) est peu prometteur: ces personnes ont la moitié moins de chances de réussir le $1^{\mathrm{er}}$ propédeutique. Quant au $2^{\mathrm{e}}$ propédeutique, jusqu'à présent seules deux personnes de la liste d'attente l'ont tenté.

- La procédure telle qu'elle est actuellement appliquée pourrait dans le pire des cas avoir pour conséquence que la durée des études ne se raccourcisse que lentement et que le nombre d'abandons et la répartition des notes ne changent guère par rapport à la situation prévalant avant l'introduction du NC. Il faudrait alors s'interroger sur l'effet dissuasif que l'on a toujours voulu donner jusqu'ici au test et sur l'information dispensée sur les conditions dans lesquelles se déroulent les études et s'exerce la profession pour savoir si le test n'aurait pas d'effets sélectifs indésirables.

Quelles sont les possibilités d'optimiser le système afin de rehausser la proportion des personnes aptes dans les études de médecine, et parlà d'optimiser l'utilisation des ressources?

- Influencer les candidatures en s'adressant davantage aux personnes aptes. Il faudrait analyser si les facteurs de dissuasion «voulus»NC et test d'aptitudes - et les moins bonnes perspectives professionnelles («pléthore» de médecins, régularisation des coûts, etc.) ne dissuadent pas davantage les plus aptes à faire acte de candidature. Corrélativement, il faudrait se demander pourquoi le nombre des candidatures masculines est si bas et diminue chaque année. Cette analyse n'aurait aucune incidence sur l'égalité des chances. Un résultat important et encourageant de cette évaluation est que pour un même test, donc pour une même aptitude, les femmes ont pratiquement les mêmes chances que les hommes de réussir les examens en un temps optimal et avec de bonnes notes. 
- Renoncer à admettre des personnes dont il est prouvé qu'elles n'ont pas atteint un «niveau minimal» d'aptitudes, soit un niveau où l'on sait d'entrée de jeu que les chances de réussir l'examen sont faibles. Il faudrait alors accepter de ne pas exploiter entièrement les capacités fixées (les capacités actuelles des deux premières années comportent encore des estimations relatives aux retraits, abandons et retards, qui reposent sur des chiffres antérieures au NC).

- Engager une vaste réforme des filières, afin de créer des conditions d'études telles que les personnes aptes puissent optimiser la durée et la qualité de leurs études et que les abandons deviennent enfin l'exception.

\section{Références}

1 Hänsgen K-D, Spicher B. Zwei Jahre Numerus Clausus und Eignungstest für das Medizinstudium in der Schweiz (EMS). Teil 1: Erfahrungen mit dem EMS als Zulassungskriterium. Schweiz Ärztezeitung 2000;81(12):666-72.

2 Hänsgen K-D, Spicher B. Zwei Jahre Numerus Clausus und Eignungstest für das Medizinstudium in der Schweiz (EMS). Teil 2: EMS und Chancengleichheit. Schweiz Ärztezeitung 2000;81(13): 723-30.

3 Trost G, Blum F, Fay E, Klieme E, Maichle U, Meyer M, Nauels H-U. Evaluation des Tests für Medizinische Studiengänge (TMS): Synopse der Ergebnisse. Bonn: ITB; 1998.

4 Hänsgen K-D, Spicher B. EMS Eignungstest für das Medizinstudium in der Schweiz. Bericht über die Durchführung und Ergebnisse, Evaluation der Vorhersage von Prüfungserfolg - avec un résumé en français. Berichte des Zentrums für Testentwicklung und Diagnostik Band 7. Fribourg; 2001. Accessible sur Internet: www.unifr.ch/ztd/EMS. 